\title{
Iterative Design Process For The Development And Testing Of Cooperative Applications
}

Tobias Lorenz 


\section{Content}

$>$ Short Introduction

7 German Aerospace Center (DLR)

7 Institute of Transportation Systems

7 Iterative Design Process

7 Overview

7 Model Selection

7 Model Parameterization

$>$ Simulation / Evaluation

$>$ Conclusion 


\section{Short Introduction - DLR and Institute TS}




\section{German Aerospace Center}

Areas of Research

$>$ Aeronautics

7 Space

$>$ Transport

$>$ Energy
DLR in numbers

7 Budget:

2006 1.168 M Euro

2007 1.224 M Euro

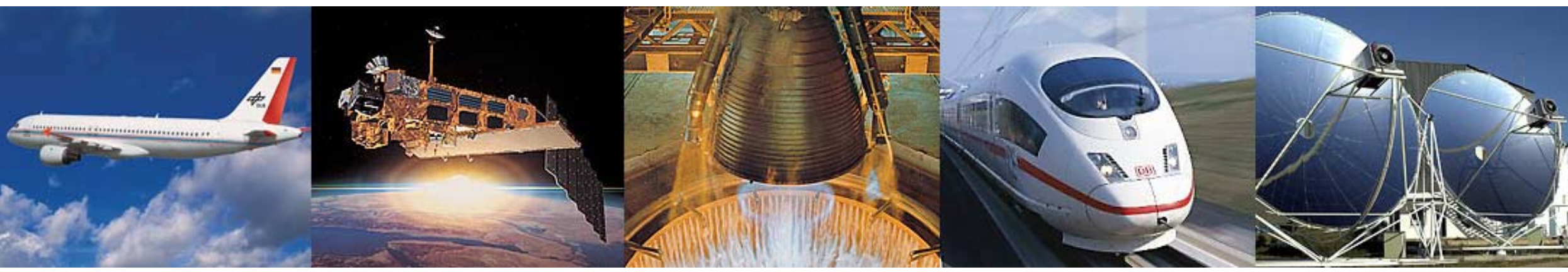




\section{Locations and Employees}

5.600 employees work at 28 research institutes and facilities at 13 locations.

Offices in Brussels, Paris and Washington.

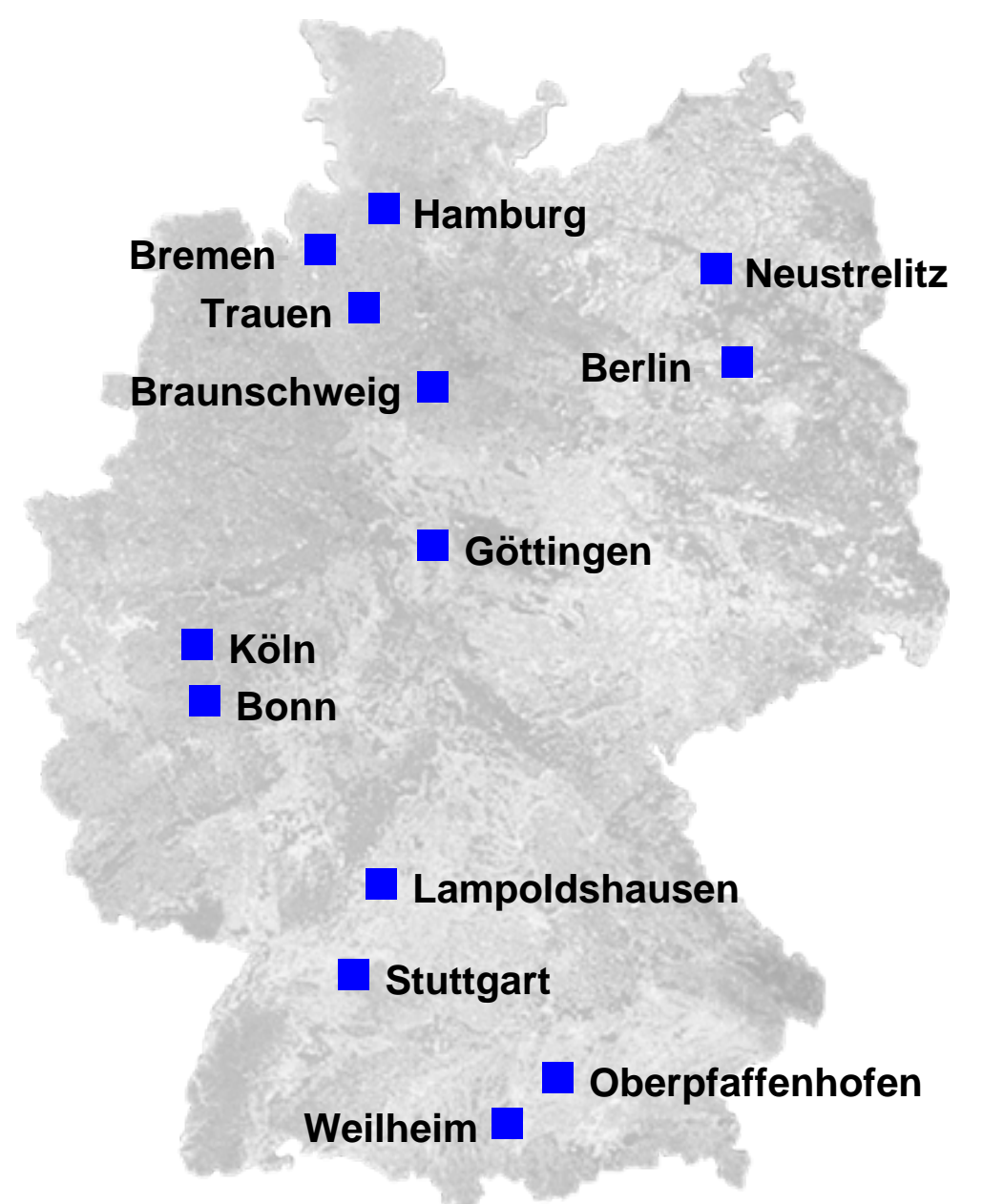




\section{Transportation \\ Participating institutes}

$>$ Institute of Transport Research

$>$ Project Transport Studies

7 Institute of Transportation Systems

7 Institute of Vehicle Concepts

... and 21 more institutes from aeronautics, space and energy

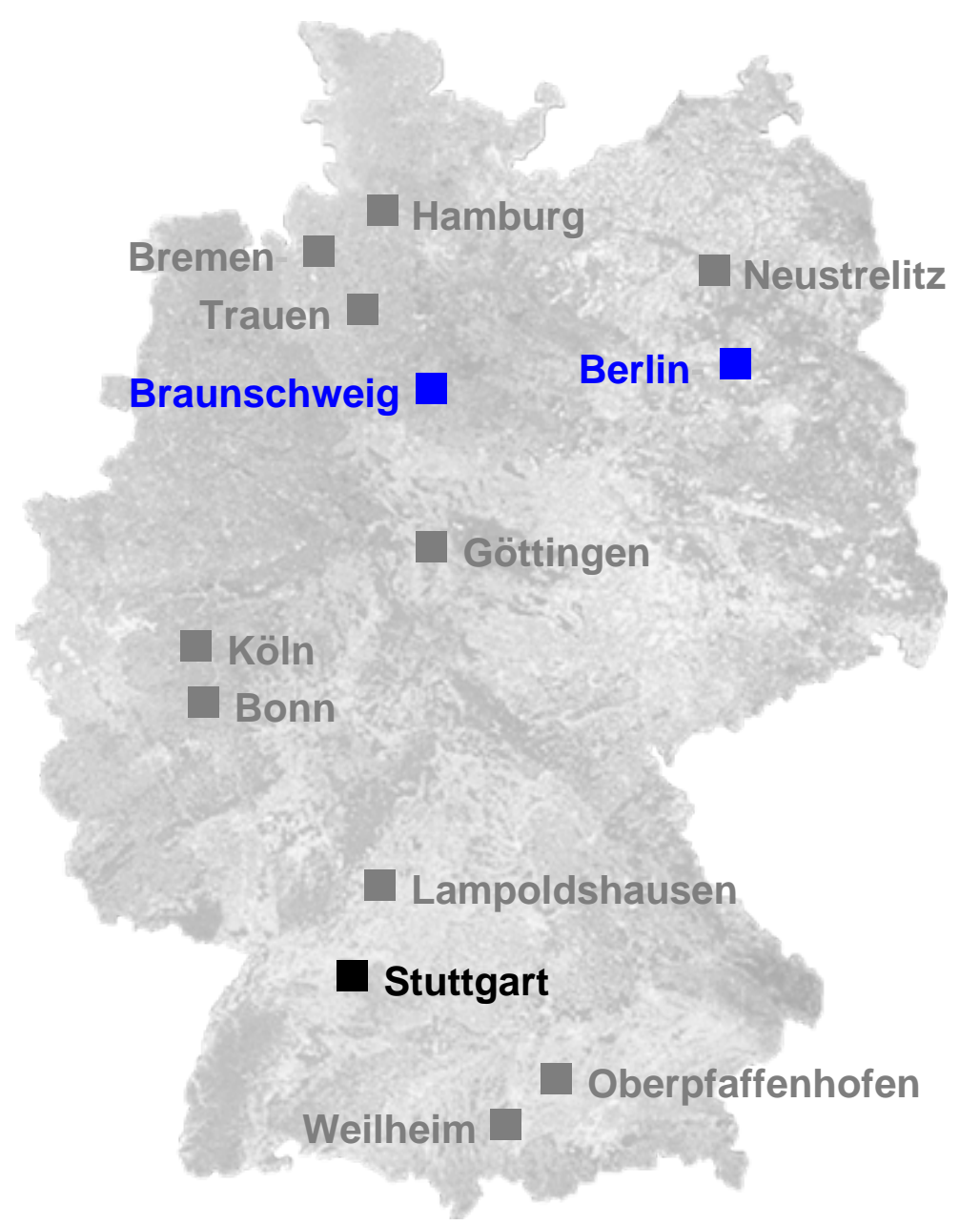




\section{Institute of Transportation Systems}

Residence:

Since:

Director:

Employees:
Braunschweig and Berlin

March 2001

Prof. Dr.-Ing. Karsten Lemmer

Presently 100 employees

from various scientific disciplines

Range of tasks

$>$ Basic research

$>$ Creating concepts and strategies

$>$ Prototype development

Fields of Research

$>$ Automotive

$>$ Railway Systems

$>$ Traffic Management 


\section{Iterative Design Process}

Overview 


\section{Iterative Design Process Introduction and Motivation}

$>$ Increasing importance of conducting simulation tests during the development and evaluation process because

7 Basically field operational tests (FOTs) are applicable but

$>$ Large number of equipped vehicles

7 Huge testing areas especially urban areas required

7 Immense costs

7 Influence of different penetration rates

$>$ To run these simulation tests systematically by using the right simulators an iterative design process including guidelines and code of practice is indispensible 


\section{Iterative Design Process}

Overview

7 Different steps within this process

$>$ Accident analysis provides an application idea

$>$ Study statistics on road accidents

7 Reasons for the accidents

7 Development and testing of the application within different scenario views

$>$ Model selection

$>$ Parameterization

$>$ Evaluation

7 Prototype as final outcome

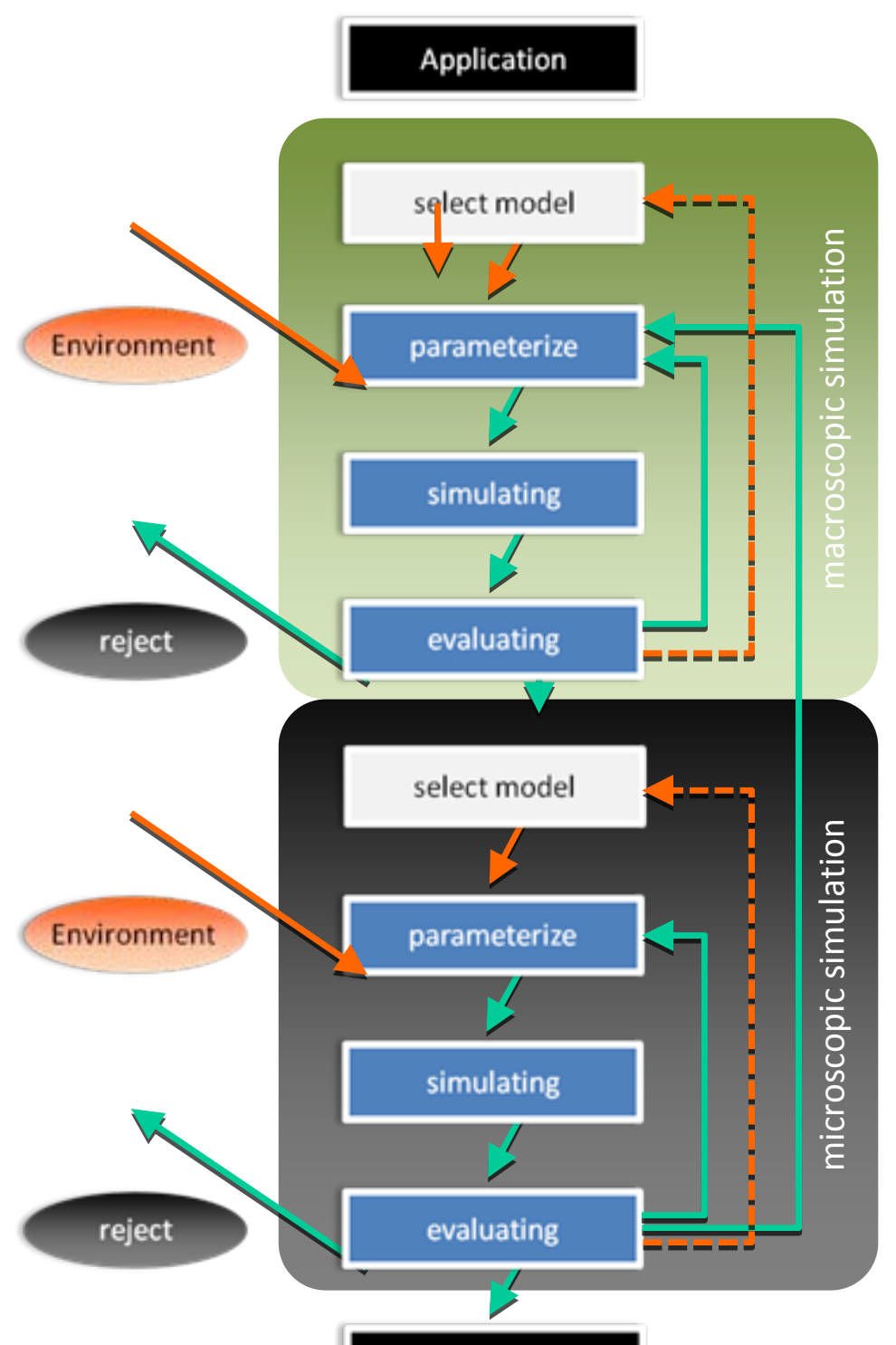

Prototype 


\section{Iterative Design Process Model Selection}




\section{Iterative Design Process}

Model Selection I - Overview

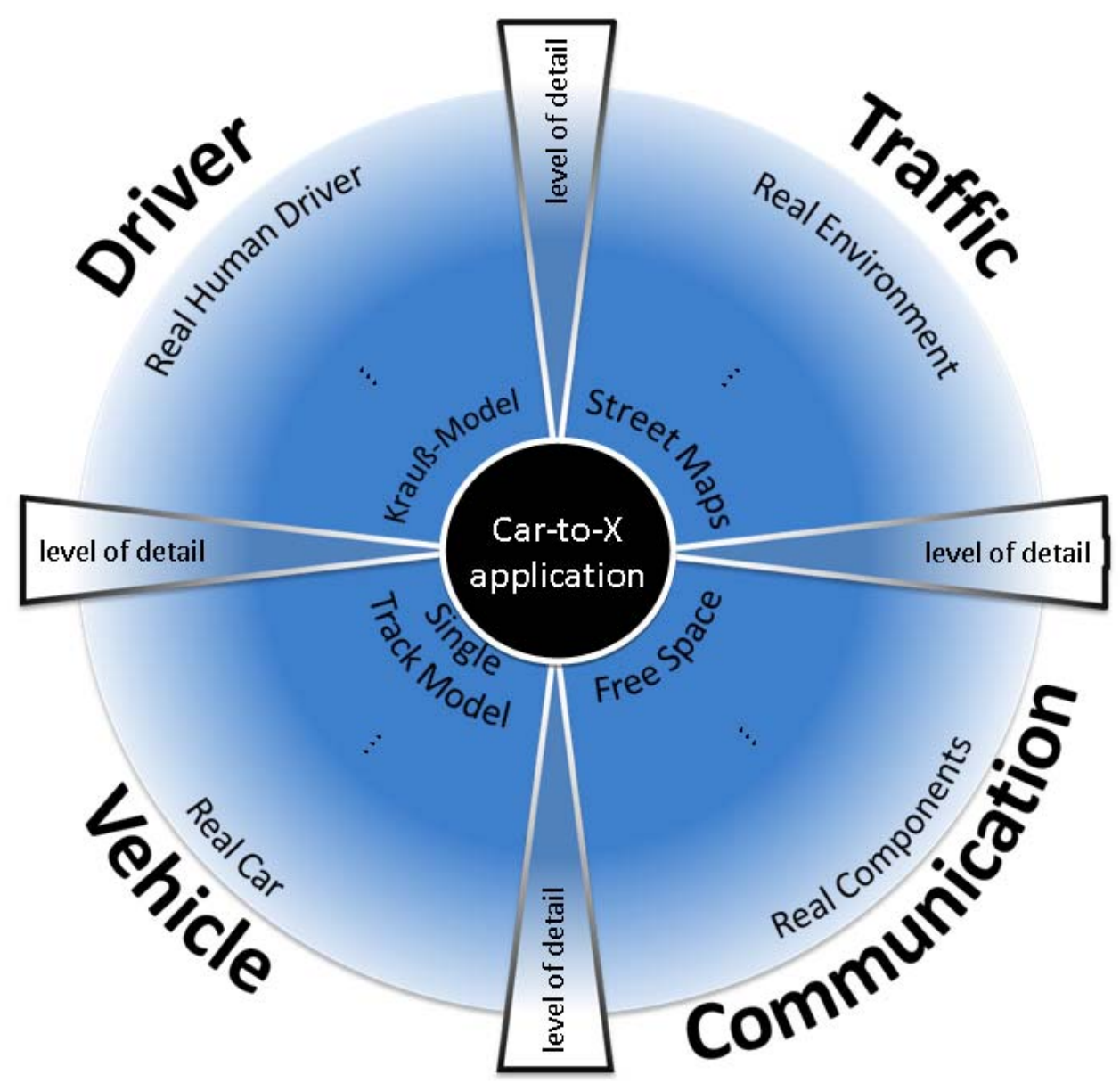




\section{Iterative Design Process Model Selection II - Background}

$>$ Often Simulators/Models are used which are not applicable for the current research question because

$>$ Too many unknown parameters within the model

$>$ Environmental influences are not considered

$>$ Non-transferability of the results to the real world

7 Example

7 Often NS-2 is used with non-deterministic communication model but

$>$ Not applicable for the investigation of safety applications in urban environments

7 Buildings and other cars are not considered for the communication 


\section{Iterative Design Process \\ Model Selection III - Key Parameters}

7 Two main key parameters for model selection

$>$ Scenario scale

$>$ Macroscopic

$>$ Traffic flows in huge areas

$>$ Traffic Management

7 Microscopic

$>$ Certain parts of the traffic flow

$>$ Safety critical applications

7 Communication aspects (e.g. message propagation)

$>$ The description of the Car-to- $X$ application

$>$ Requirements

$>$ Intended Effect

$>$ Performance Indicators

$>\quad \ldots$ 


\section{Iterative Design Process}

Model Selection IV - "Stationary Vehicle Warning"

7 Example - "Stationary Vehicle Warning" (ETSI BSA ${ }^{1}$ )

$>$ Informs the driver about a stationary vehicle

7 Safety application

$>$ Microscopic view only

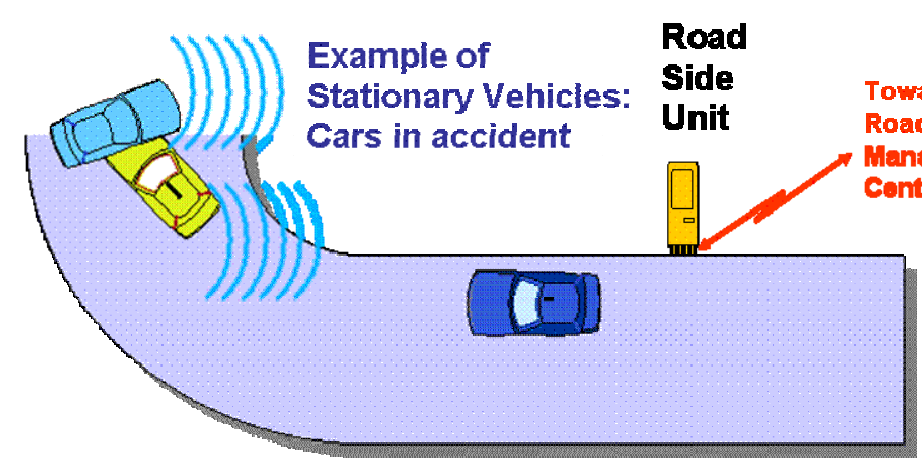

oad Traffic

Managemen

Centre

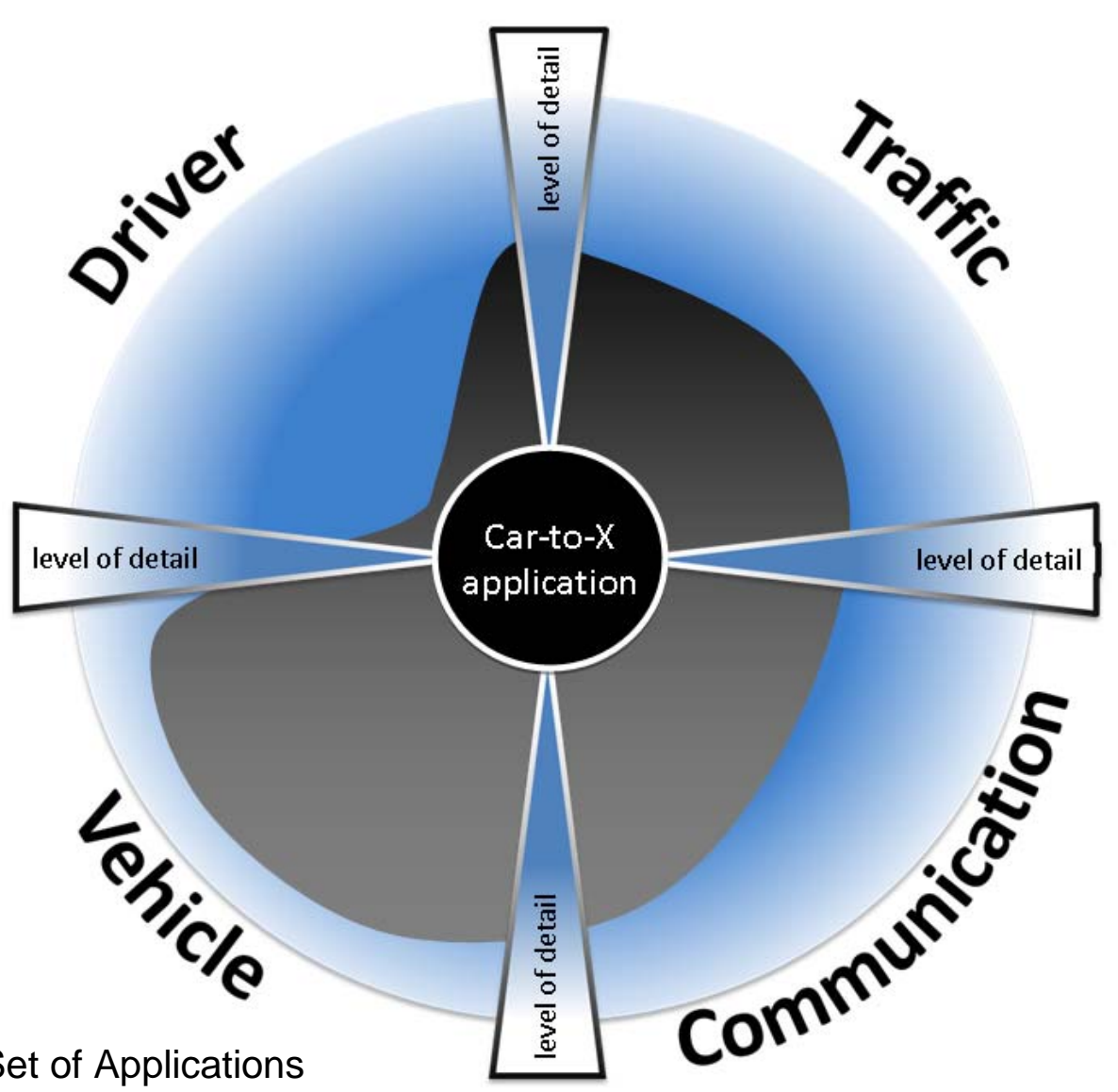




\section{Iterative Design Process}

Model Selection V - "Stationary Vehicle Warning"

7 Communication Model

$>$ Environmental influences

$>$ Deterministic message propagation

7 No simulation of ISO/OSI layers like MAC/NET/TRA

7 Latency times, Sample frequency

7 Signal Damping

$>$ Communication range

$>$ Driver Model of surrounding traffic

$>$ e.g. Krauß model

$>$ Vehicle Model of ego vehicle

$>$ Non-linear two track model

7 Traffic

> e.g. VISSIM, SUMO, ... 


\section{Iterative Design Process}

Model Selection VI - Communication Simulator

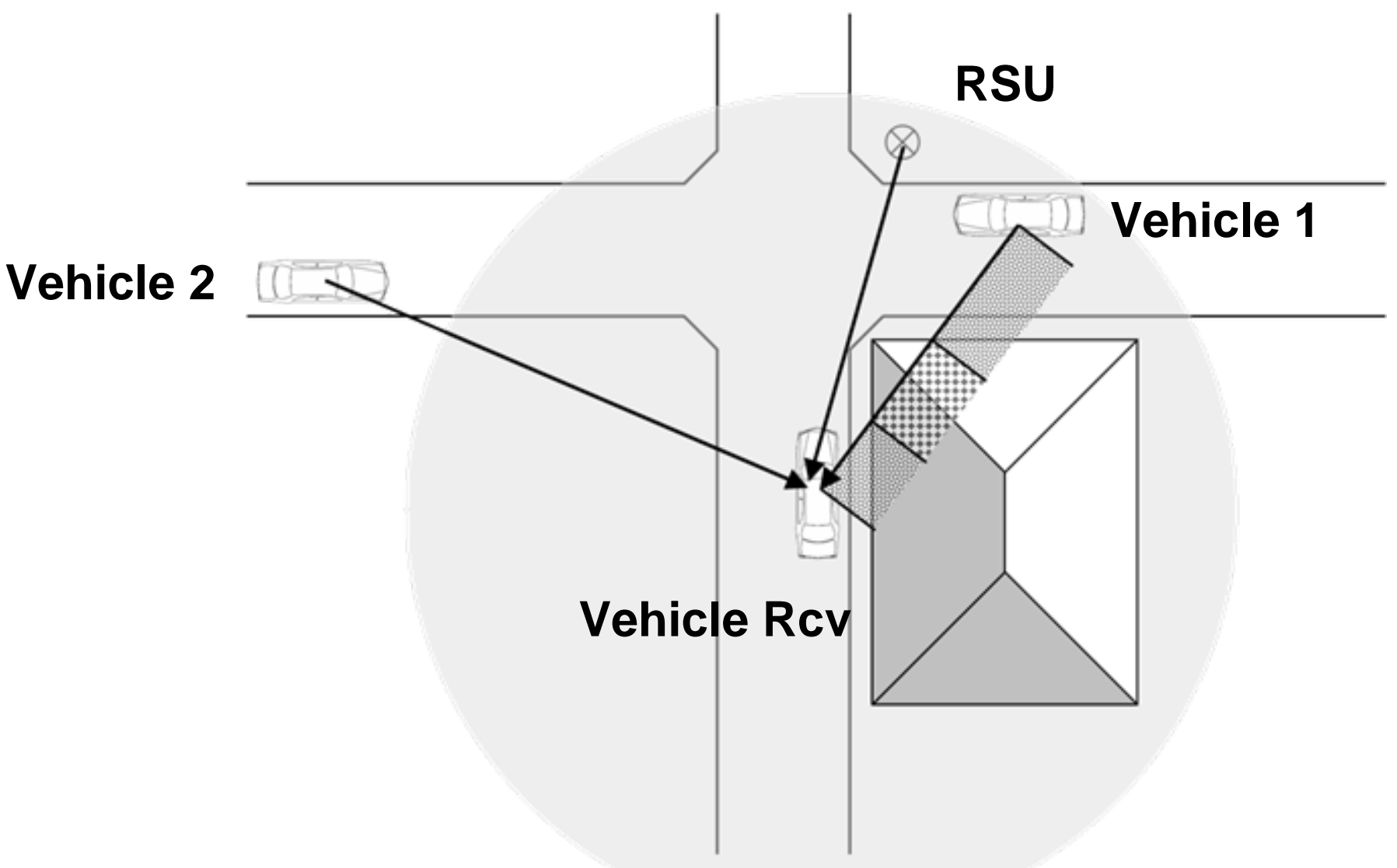





\section{Iterative Design Process \\ Model Parameterization I - Overview}

7 Different models provide different influencing parameters

7 Some of the parameters have to be varied and some are fixed within the simulation

7 Static parameters

7 Variable parameters

$>$ Variable parameters are varied until the intended effect of the application can be verified

7 Traffic management

7 Macroscopic scenario view

$>$ e.g. identification of penetration rate and needed communication range to achieve the intended effect

$>$ Microscopic scenario view

$>$ Validation of the identified parameters by considering environmental influences, too 


\section{Iterative Design Process}

Model Parameterization II - "Stationary Vehicle Warning"

$>$ Stationary Vehicle Warning - Safety application

$>$ One aim could be the investigation of communication aspects and their influence on driver behavior

$>$ Intended effect

7 e.g. warn driver $300 \mathrm{~m}$ before traffic obstruction

$>$ Static parameters

7 Properties of environmental surroundings

7 Communication frequency $5.9 \mathrm{GHz}$

7 Variable Parameters

7 Transmitting power

$>$ Antenna position 



\section{Iterative Design Process}

Simulation/Evaluation - "Stationary Vehicle Warning"

$>$ For the simulation trials

$>$ The static parameters are fixed

$>$ The variable parameters will be varied

$>$ Iterative process

7 Fix all variable parameters but one

7 Vary the one left

$>$ If the intended effect is not verifiable change one or more of the fixed parameters and restart process

7 Example

7 Fix antenna position

$>$ Vary the transmitting power 


\section{Iterative Design Process}

Conclusion 


\section{Iterative Design Process Conclusion}

$>$ The iterative design process emphasize the importance of simulation in the area of Car-to- $X$ systems

7 Selection of the right simulator models is indispensible

7 For Car-to-X the focus is on communication simulation

$>$ Choice of the right model parameters is important

$>$ When running the iterative design process successfully for simulation it can be adapted to FOT

$>$ There the model selection process is omitted 


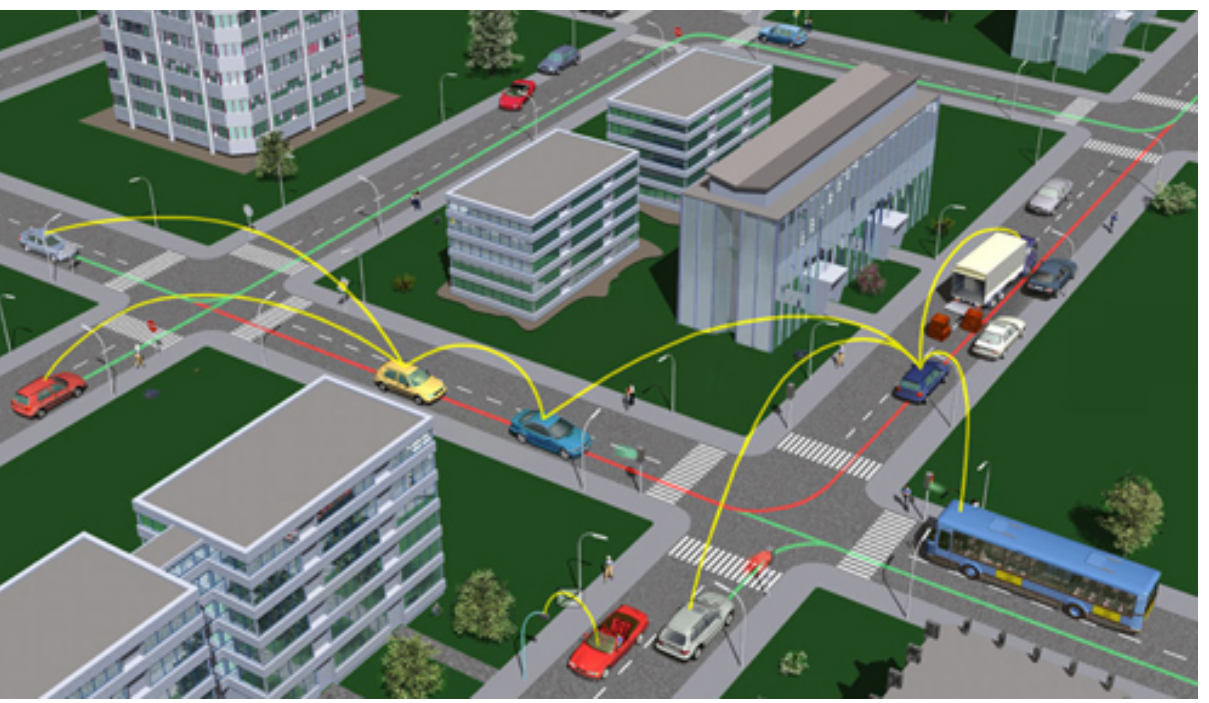

7 Tobias Lorenz

Institute of Transportation Systems

Lilienthalplatz 7

38108 Braunschweig

$>\quad+49531295-3475$

> Tobias.Lorenz@dlr.de 